\title{
Condiciones sociales de los pobladores en las zonas de conflicto minero en el Perú
}

\section{Social conditions of the peoples in the mining conflict areas in Peru}

\author{
Máximo Ramírez, Mauro Estrada, Rodolfo Ramirez 3
}

\section{RESUMEN}

El objetivo de esta investigación fue describir y analizar las condiciones sociales de los pobladores en las regiones del Perú, donde se suscitan conflictos sobre la explotación minera. Los resultados demuestran el desconocimiento de los pobladores en relación a las normas legales, de los pueblos que conforman las zonas mineras.

El Perú, es uno de los países más importantes de Latinoamérica en cuanto a la minería, empero encuentra sumergido en conflictos sociales muy sensibles, hasta el extremo de ponerse en riesgo en cuanto a su desarrollo económico. Las conclusiones indican que las políticas de estado, no han sido aplicadas adecuadamente como mecanismos de diálogo al apego de las normas constitucionales, se han dejado marginadas las políticas institucionales, que pudieron haber servido para el aprovechamiento de los recursos naturales con desarrollo sustentable, asumiendo con responsabilidad los derechos de los pueblos. En los lugares donde existen conflictos relacionados a la minería, los ambientes sociales están polarizados, manipulados con mucha habilidad por los agitadores ideológicos, quienes buscan desestabilizar todo esfuerzo de tolerancia entre los responsables autoridades y los pobladores.

Las evidencias muestran ausencia de dialogo entre los pobladores, autoridades locales y el gobierno central, ausencia absoluta de los colegios profesionales de ingenieros, de abogados, de médicos entre otros. Finalmente, es necesario analizar la Carta Magna del Perú, que precisa la propiedad estatal del subsuelo, que señala en su artículo $66^{\circ}$ dice literalmente "Los recursos naturales, renovables y no renovables, íntegramente pertenecen al patrimonio de la nación.

Palabra clave: Condiciones sociales de los pobladores en las zonas de conflicto

1. Doctor en Educación Licenciado en Educación, Maestro en docencia Universitaria y Gestión Educativa, Doctor en Educación y administración. Email: epg_uap@hotmail.com

2. Abogado, Maestro en Derecho Civil, Doctor en Educación y Derecho. Email: estrada_mauro2208@hotmail.com

3. Licenciado en Periodismo, Maestro en Ministración y Dirección de Empresas, Doctor en Educación. Email: rmramirezc@hotmail.com 


\section{ABSTRACT}

The research work aimed to describe and analyze social conditions of the peoples in the areas of mining conflict in Peru. The results show that there is ignorance of current legal norms of the towns that make up the areas of mining influence. Peru, despite being one of the most important countries in Latin America in terms of mining, is plunged into one of the most sensitive and sensitive social conflicts, to the point of putting its economic growth at risk.

The conclusions indicate that state policies have failed to apply the mechanisms of dialogue for the application of constitutional norms that could be used for the use of natural resources with sustainable development, assuming responsibility for the rights of peoples. In the areas of mining conflict, the social environment is polarized, skillfully used by the ideological agitators, who seek to destabilize all efforts of coexistence and tolerance between authorities and the settlers.

There is also evidence of absence of dialogue between residents, local authorities and the central government, absolute absence of professional associations such as: engineering school, law school, among others. Finally, it is necessary to analyze the Political Constitution of Peru, which specifies the criteria of state property of the subsoil, which establishes in its article 66 that "Natural resources, renewable and non-renewable, are the patrimony of the nation.

Key word: Social conditions of peoples in conflict zones

\section{INTRODUCCION}

El propósito de esta investigación, fue describir y analizar las condiciones sociales de pobladores en las regiones del Perú, donde existen conflictos a causa de la explotación minera. En razón de ello, el trabajo se torna interesante porque se pretende conocer las implicancias del derecho ambiental en cuanto al uso de recursos naturales y sus consecuencias en las problemáticas sociales.

La problemática social existente en zonas de afluencia minera, ocurren en diferentes países, empero en el Perú, esta problemática es latente y va en aumento, ya que los partidos políticos y las ONGs, por querer sembrar sus ideológicas, están creando desintegración entre la sociedad y sus autoridades aprovechando la coyuntura.

Según los especialistas, el Perú es el segundo país minero más importante en cuanto a las normas de protección ambiental se refiere, que en últimos años, ha crecido exponencialmente, recibiendo flujos de inversión, por US\$ 51,000 millones (fuente diario el comercio).
Por problemas sociales existentes, las inversiones mineras se encuentran en situación de riesgo, no existe capacidad de respuesta del estado, menos de las empresas mineras para buscar puntos de concordancia para solucionar los conflictos sociales.

Más allá de las agendas propias de los partidos políticos que buscan réditos y las ONGs, de tendencia izquierdista, el estado debe propiciar el dialogo con el pueblo, con la asesoría de las universidades públicas y privadas, para analizar los puntos discordante en marco del derecho ambiental. Se debe aspirar a una actividad minera responsable, que sirva como apalancamiento para desarrollo sostenible de los pobladores colindantes con la minería.

La mesa de trabajo debe ser permanente con agendas de educacion, trabajo y salud, deben estar compuestos por todos los representantes sociales, no solo los representantes que en muchos casos tienen sesgos ideológicos. 
Las normas vigentes relacionadas a las políticas medioambientales se encuentran enmarcadas en la carta magna del Perú, que regula las reglas de juego en cuanto al derecho ambiental, en cuanto se analice de manera consiente y profesional, se pueda hacer posible su sostenibilidad y su convergencia con las inversiones en todos los sectores extractivos incluido el sector minero, con la participación de los pobladores en la planificación, se podrá lograr un desarrollo sostenible de los proyectos, que respete los derechos de las comunidades, que propicie el empoderamiento de los pobladores responsables del futuro de sus pueblos, con reembolso de fondos por la actividad minera reinvertirlos en educacion y salud, de sus comunidades con lo cual sería satisfecha la necesidad de los pueblos.

El derecho ambiental respecto a la explotación minera, debe ser claro estableciendo condiciones sociales favorables que propicie políticas de educacion y desarrollo, desterrando situaciones de conflictos absurdos entre coetáneos.

Las opciones ideológicas y los apetitos particulares de quienes participan en las negociaciones, no deben seguir constituyendo más situaciones de conflicto entre los pobladores. No deben seguir generando tensiones que originan protestas, enfrentamientos entre pueblos vecinos, convirtiéndose en delitos que desbordan las normas de convivencia social, creando tensión innecesariamente.

Por desconocimiento a las leyes nacionales, los pobladores atentan contra los derechos fundamentales de otros pueblos, bloqueando vías de comunicación, donde el estado ha perdido el principio de autoridad, por lo urge participación de colegios profesionales, de organizaciones encargadas en resolución de conflictos, el Estado debe propiciar diálogos abiertos con los pobladores para reestablecer principio de autoridad sin la manipulación de agentes externos que solo buscan beneficios particulares.

En los últimos años, los problemas respecto a la explotación minera se ha polarizado, con razón o sin ella, han sido abordados diferentes escenarios, al verse afectados los derechos colectivos de los pueblos, se reclama mayor protección al ecosistema y respeto a los derechos humanos.

En una sociedad extremadamente individualista, resulta difícil entender que exista derechos colectivos, que el Estado considere que los derechos de los pobladores es una prioridad, políticamente esto sería correcta, ya de este modo se podría ponderar la protección, a los derechos, como producto de las negociaciones políticas jurídicos.

Las normas constitucionales respecto al derecho ambiental, deben propiciar la participación de las regiones involucradas, propiciar en las comunidades el derecho a participar en la meza de diálogo para superar los retos en favor de los pueblos menos favorecidos.

El derecho minero dentro del marco constitucional, debe estar abierto al dialogo entre las comunidades y sus autoridades, generar espacios para que no sigan siendo caldo de cultivo de los agitadores sociales, epicentro de conflictos amañados por las ONGs, las protestas anti mineras fabricadas desde el exterior.

Para reestablecer las protestas contra las decisiones del central, no basta aumentar el presupuesto del canon minero, propiciar una que favorezca la distribución de ingresos producto de la actividad minera en el país. Si no, se requiere presencia permanente del estado en áreas de educacion, salud y trabajo en las regiones para forjar una nueva cultura.

Conforme reporta la Defensoría del Pueblo, en el Perú existen un total de 214 problemas sociales a nivel nacional, de los cuales 192 son problemas sociales y ambientales. Amazonas con 3, Áncash con 23, Apurímac con 24, Arequipa con 8, Ayacucho con 12, Cajamarca con 14, Cusco con 11, Huancavelica con 2, Huánuco con 1, Ica con 2, Junín con 14, La Libertad con 5, Lambayeque con 3, Lima Metropolitana con 1, Lima provincias con 8, Loreto con 11, Madre de Dios 
con 1, Moquegua con 2, Pasco con 4, Piura con 13, Puno con 18, San Martín con 2, Tacna con 5, Tumbes con 4 y Ucayali con 1.

Los problemas en su mayoría, son manejados por agitadores sociales que aprovechan la ausencia del estado, los conflictos son manejados políticamente, los problemas ambientales solo son pretextos, no salvaguardan los derechos de los pueblos,

Los problemas sociales sobre la explotación minera, podrían solucionarse con la correcta aplicación del derecho ambiental, Ley de Consulta Previa, que aplicadas adecuadamente evitaría la manipulación de los pueblos por los advenedizos, como ha venido ocurriendo últimamente.

\section{MATERIAL Y MÉTODOS}

Para desarrollar el trabajo de investigación se utilizaron textos especializados, audios, videos, pizarras, multimedia, materiales de reciclaje, papel bond, mobiliarios varios.

Se realizó una investigación descriptiva, dado que este método permitió la descripción de los diversos aspectos características de los problemas planteados, también permitió analizar las teorías y principios relacionados a las condiciones sociales de los pueblos comprendidos en las zonas mineras en el Perú, que ha permitido analizar desmembrando de un todo, descomponiéndolo en sus partes observando los hechos, asimismo permite conocer las doctrinas internacionales y nacionales relativas a la investigación científica que fueron explicados objetivamente relacionando, sus efectos y consecuencias.

Para realizar el trabajo de campo, se utilizó el diseño trasversal, porque permitió determinar los beneficios y limitaciones del problema planteado, permitiendo involucrar a la población de estudio permitiendo observar individualmente y en grupos.

\section{RESULTADOS}

Como estrategia de trabajo, se codificó los resultados de las encuestas relacionadas con la investigación, los datos fueron categorizados en orden consecutivo para obtener los resultados. Se entrevistaron a las personas involucradas con los problemas de explotación minera.

\section{Tabla $\mathbf{N}^{\circ} 1$}

Sobre la pregunta ¿Qué avances muestra el derecho constitucional en el tratamiento de los recursos naturales en las zonas mineras? la tabla muestra que El 8.2\% de encuestados responden que el derecho constitucional ha avanzado totalmente. El $24.6 \%$ de los encuestados responden que avanzado regularmente. El 24.6\% de los encuestados responden que avanzado. El 9.8\% de los encuestados responden que no es prioritario. Y el 32.8\% de los encuestados responden que no existe ningún tipo de avances.

$\begin{array}{ccc}\begin{array}{c}\text { ¿Qué avances muestra el derecho constitucional en el } \\ \text { tratamiento de los recursos naturales en las zonas } \\ \text { mineras? }\end{array} & \mathrm{N}^{\mathrm{o}} & \% \\ \text { Avanzado Totalmente } & 10 & 8.20 \% \\ \text { Avanzado regularmente } & 30 & 24.60 \% \\ \text { Avanzado } & 30 & 24.60 \% \\ \text { Ningún tipo de avance } & 40 & 32.80 \% \\ \text { No es prioritario } & 12 & 9.80 \% \\ \text { TOTAL } & 122 & 100 \%\end{array}$




\section{Tabla $\mathrm{N}^{\circ} 2$}

Sobre la pregunta ¿Cómo se regularía la explotación de las tierras comunales en zonas de conflicto? tabla muestra que $\mathrm{El}$ 65.6\% de los encuestados responden que se regularía mediante la reforma constitucional. El 8.2\% de los encuestados responden que con el retorno a la constitución del 79. El $14.7 \%$ de los encuestados respondieron que con la formulación de proyectos de ley. Y el $11.5 \%$ de los encuestados respondieron que regulando los derechos de los pueblos

$\begin{array}{ccc}\begin{array}{c}\text { ¿Qué avances muestra el derecho constitucional en el } \\ \text { tratamiento de los recursos naturales en las zonas } \\ \text { mineras? }\end{array} & \text { No } & \% \\ \text { Avanzado Totalmente } & 10 & 8.20 \% \\ \text { Avanzado regularmente } & 30 & 24.60 \% \\ \text { Avanzado } & 30 & 24.60 \% \\ \text { Ningún tipo de avance } & 40 & 32.80 \% \\ \text { No es prioritario } & 12 & 9.80 \% \\ \text { TOTAL } & 122 & 100 \%\end{array}$

\section{Tabla $\mathrm{N}^{\circ} 3$}

Sobre la pregunta ¿Cómo se puede normar el derecho constitucional para lograr el dialogo entre las autoridades y pobladores? la tabla muestra que El 49.2\% de los encuestados responden que se podría normar realizando nuevas leyes. El 8.2\% de los encuestados responden que elaborando nuevos decretos legislativos. El 23\% de los encuestados responden que coordinando con los pobladores. El 8.2\% de los encuestados responden que imponiendo nuevas normas legales. Y el 11.4\% de los encuestados respondieron que realizando capacitaciones permanentes.

$\begin{array}{ccc}\begin{array}{ccc}\text { ¿Cómo se puede normar el derecho constitucional para } \\ \text { lograr el dialogo entre las autoridades y pobladores? }\end{array} & \mathrm{N}^{\mathrm{o}} & \% \\ \text { Realizando nuevas leyes. } & 60 & 49.20 \% \\ \text { Elaborando nuevos decretos Legislativos. } & 10 & 8.20 \% \\ \text { Coordinando con pobladores. } & 28 & 23 \% \\ \text { Imponiendo nuevas normas legales. } & 10 & 8.20 \% \\ \text { Realizando capacitaciones permanentes } & 14 & 11.40 \% \\ \text { TOTAL } & 122 & 100 \%\end{array}$

\section{Tabla $N^{\circ} 4$}

Sobre la pregunta ¿Por qué existen discrepancias en la aplicación de normas entre los organismos gubernamentales sobre las tierras con recursos mineros? la tabla muestra que El 16.4\% de los encuestados responden que existen discrepancias por desconocimiento a las normas. El $41 \%$ de los encuestados responden porque no existen normas jurídicas. El 32.8\% de los encuestados responden que son controversiales. Y el 9.8\% de los encuestados responden que son inadecuados. 


$\begin{array}{ccc}\begin{array}{c}\text { ¿Por qué existen discrepancias en la aplicación de } \\ \text { normas entre los organismos gubernamentales sobre } \\ \text { las tierras con recursos mineros? }\end{array} & \text { No } & \% \\ \text { Por desconocimiento } & 20 & 16.40 \% \\ \text { Por no existir normas jurídicas } & 50 & 41 \% \\ \text { Por ser controversiales } & 40 & 32.80 \% \\ \text { Por ser Inadecuados } & 12 & 9.80 \% \\ \text { Otros } & 0 & 0 \% \\ \text { TOTAL } & 122 & 100 \%\end{array}$

\section{Tabla $\mathrm{N}^{\circ} 5$}

Sobre la propuesta, Marque las siguientes alternativas sobre las normas que usted conoce en la solución de conflictos entre comuneros y autoridades. La tabla muestra que El 8.2\% de los encuestados responden que jurisprudencias constitucionales. El 16.4\% de los encuestados responden que derechos de territoriales. $16.4 \%$ de los encuestados responden que gestiones ambientales. El $49.2 \%$ de los encuestados responden que derechos sociales. Y el 9.8\% de los encuestados responden que las reformas agrarias.

$\begin{array}{ccc}\begin{array}{c}\text { Marque las siguientes alternativas sobre las normas } \\ \text { que usted conoce en la solución de conflictos entre } \\ \text { comuneros y autoridades }\end{array} & \mathrm{N}^{\mathrm{o}} & \% \\ \text { Jurisprudencia Constitucional. } & 10 & 8.20 \% \\ \text { Derechos Territoriales. } & 20 & 16.40 \% \\ \text { Gestiones ambientales. } & 20 & 16.40 \% \\ \text { Derechos sociales. } & 60 & 49.20 \% \\ \text { Reformas agrarias } & 12 & 9.80 \% \\ \text { TOTAL } & 122 & 100 \%\end{array}$

\section{Tabla $\mathrm{N}^{\circ} 6$}

Sobre la pregunta ¿A qué se deben las carencias de los funcionarios públicos y los magistrados del poder judicial para acabar con los conflictos sociales? la muestra que El 8.2\% de los encuestados responden que principio de respeto al territorio ancestral de los pueblos indígenas. El $24.6 \%$ de los encuestados responden que principio de inviolabilidad de los derechos humanos. El $41 \%$ de los encuestados responden que principios rectores del derecho a la consulta. El 8.2\% de los encuestados responden que principio de dignidad. Y el 18\% de los encuestados responden que acciones políticas generales.

$\begin{array}{ccc}\text { ¿A qué se deben las carencias de los funcionarios } & & \\ \text { públicos y los magistrados del poder judicial para } & \mathrm{N}^{\mathrm{o}} & \% \\ \text { acabar con los conflictos sociales? } & & \\ \text { Respeto al territorio de los pueblos } & 10 & 8.20 \% \\ \text { Respeto a los Derechos Humanos } & 30 & 24.60 \% \\ \text { Respeto a rectores del derecho a la consulta. } & 50 & 41 \% \\ \text { Respeto a la Dignidad } & 10 & 8.20 \% \\ \text { Acciones políticas generales } & 22 & 18 \% \\ \text { TOTAL } & 122 & 100 \%\end{array}$




\section{Tabla $\mathbf{N}^{\circ} 7$}

Sobre la pregunta ¿De qué manera se puede propiciar el dialogo acerca de la promoción de tierras comunales entre las comunidades y el estado? la tabla muestra que El 30.3\% de los encuestados responden que la constitución lo permite. El 8.2\% de los encuestados responden que el gobierno hace lo correcto. El 27.1\% de los encuestados responden que el gobierno no hace lo correcto. Y el $34.4 \%$ de los encuestados responden que se viola los derechos de las comunidades.

$\begin{array}{ccc}\begin{array}{c}\text { ¿De qué manera se puede propiciar el dialogo acerca } \\ \text { de la promoción de tierras comunales entre las }\end{array} & \mathrm{N}^{\mathrm{o}} & \% \\ \text { comunidades y el estado? } & & \\ \text { Con la constitución. } & 37 & 30.30 \% \\ \text { Con gobierno dialogante } & 10 & 8.20 \% \\ \text { El gobierno no hace correctamente } & 33 & 27.10 \% \\ \text { Violan derechos de comunidades } & 42 & 34.40 \% \\ \text { otros } & 0 & 0 \% \\ \text { TOTAL } & 122 & 100 \%\end{array}$

\section{Tabla $\mathrm{N}^{\circ} 8$}

Sobre la pregunta ¿Cómo califica usted el Convenio 169 de la OIT, sobre la Declaración de los Derechos de los Pueblos para la explotación de los recursos naturales en tierras comunales? la tabla muestra que El 9.1\% de los encuestados responden que se cumple totalmente. El 8.2\% de los encuestados responden que se cumple mayoritariamente. El 17.2\% de los encuestados responden se cumple regularmente. El 31.1\% de los encuestados responden que se cumple minoritariamente. Y el 34.4\% de los encuestados responden que no se aplica.

\begin{tabular}{|c|c|c|}
\hline $\begin{array}{c}\text { ¿Cómo califica usted el Convenio } 169 \text { de la OIT, sobre } \\
\text { la Declaración de los Derechos de los Pueblos para la } \\
\text { explotación de los recursos naturales en tierras } \\
\text { comunales? }\end{array}$ & $\mathrm{N}^{\mathrm{o}}$ & $\%$ \\
\hline Cumplen Totalmente. & 11 & $9.10 \%$ \\
\hline Cumplen Mayoritariamente. & 10 & $8.20 \%$ \\
\hline Cumplen Regularmente. & 21 & $17.20 \%$ \\
\hline Cumplen Minoritariamente. & 38 & $31.10 \%$ \\
\hline No aplican & 42 & $34.40 \%$ \\
\hline TOTAL & 122 & $100 \%$ \\
\hline
\end{tabular}

\section{Tabla $\mathbf{N}^{\circ} 9$}

Sobre la pregunta ¿Cuáles son las razones del incumplimiento del Convenio 169 de la OIT, para con los Pueblos en las zonas en conflicto? El 29.5\% de los encuestados respondieron que por desconocimiento. El 23\% de los encuestados responden porque genera conflictos. El 21.3\% de los encuestados responden que es por controversia. El $24.6 \%$ de los encuestados responden porque es inadecuado. Y el $1.6 \%$ de los encuestados responden que otros. 


$\begin{array}{ccc}\begin{array}{c}\text { ¿Cuáles son las razones del incumplimiento del } \\ \text { Convenio } 169 \text { de la OIT, para con los Pueblos en las } \\ \text { zonas en conflicto? }\end{array} & \mathrm{N}^{\mathrm{o}} & \% \\ \text { Por falta de conocimiento. } & 36 & 29.50 \% \\ \text { Porque generan conflictos. } & 28 & 23 \% \\ \text { Es controversial. } & 26 & 21.30 \% \\ \text { Son inadecuados. } & 30 & 24.60 \% \\ \text { Otros } & 2 & 1.60 \% \\ \text { TOTAL } & 122 & 100 \%\end{array}$

\section{Tabla $\mathrm{N}^{\circ} 10$}

Sobre la pregunta ¿Cómo califica Ley de Comunidades campesinas № 24656 en cuanto a la inalienabilidad e intangibilidad de sus tierras? la tabla muestra que El 24.6\% de los encuestados responden que se cumple totalmente. El $8.2 \%$ de los encuestados responden que se cumple mayoritariamente. El $16.4 \%$ de los encuestados responden que se cumple regularmente. El 13.1\% de los encuestados responden que se cumple minoritariamente. Y el 37.7\% de los encuestados responden que no se aplica.

\begin{tabular}{|c|c|c|}
\hline $\begin{array}{l}\text { ¿Cómo califica Ley de Comunidades campesinas } \mathrm{N}^{\mathrm{O}} \\
24656 \text { en cuanto a la inalienabilidad e intangibilidad } \\
\text { de sus tierras? }\end{array}$ & $\mathrm{N}^{\mathrm{o}}$ & $\%$ \\
\hline Cumplen Totalmente. & 30 & $24.60 \%$ \\
\hline Cumplen Mayoritariamente. & 10 & $8.20 \%$ \\
\hline Cumplen Regularmente. & 20 & $16.40 \%$ \\
\hline Cumplen Minoritariamente. & 16 & $13.10 \%$ \\
\hline No aplica. & 46 & $37.70 \%$ \\
\hline TOTAL & 122 & $100 \%$ \\
\hline
\end{tabular}

\section{Tabla $\mathrm{N}^{\circ} 11$}

Sobre la pregunta ¿Cómo califica Ley de la inversión privada en el desarrollo de las actividades económicas en las zonas en conflicto? la tabla muestra que El 18.8\% de los encuestados responden que se cumple totalmente. El 26.3\% de los encuestados responden que se cumple mayoritariamente. El 34.4\% de los encuestados responden que se cumple regularmente. El 12.3\% de los encuestados responden que se cumplen minoritariamente. Y el 8.2\% de los encuestados responden que no se aplican.

$\begin{array}{ccc}\begin{array}{c}\text { ¿Cómo califica Ley de la inversión privada en el } \\ \text { desarrollo de las actividades económicas en las zonas } \\ \text { en conflicto? }\end{array} & \mathrm{N}^{\mathrm{o}} & \% \\ \text { Cumplen Totalmente. } & 23 & 18.80 \% \\ \text { Cumplen Mayoritariamente. } & 32 & 26.30 \% \\ \text { Cumplen Regularmente. } & 42 & 34.40 \% \\ \text { Cumplen Minoritariamente. } & 15 & 12.30 \% \\ \text { No aplica } & 10 & 8.20 \% \\ \text { TOTAL } & 122 & 100 \%\end{array}$




\section{Tabla $\mathrm{N}^{\circ} 12$}

Sobre la pregunta ¿Cómo califica Ley General del ambiente No 28611 en la gestión de impactos ambientales en zonas en conflicto? la tabla muestra que El 10.6\% de los encuestados responden que se cumple totalmente. El 17.2\% de los encuestados responden que se cumple mayoritariamente. El 18\% de los encuestados responden que se cumple regularmente. El 19\% de los encuestados responden que se cumple minoritariamente. Y el 35.2\% de los encuestados responden que no se aplican.

$\begin{array}{ccc}\begin{array}{c}\text { ¿Cómo califica Ley General del ambiente } \mathrm{N}^{\mathrm{o}} 28611 \text { en } \\ \text { la gestión de impactos ambientales en zonas en } \\ \text { conflicto? }\end{array} & \mathrm{N}^{\mathrm{o}} & \% \\ \text { Cumplen Totalmente } & 13 & 10.60 \% \\ \text { Cumplen Mayoritariamente } & 21 & 17.20 \% \\ \text { Cumplen Regularmente } & 22 & 18.00 \% \\ \text { Cumplen Minoritariamente } & 23 & 19 \% \\ \text { No aplica } & 43 & 35.2 \\ \text { TOTAL } & 122 & 100 \%\end{array}$

\section{DISCUSIÓN}

El Perú, durante los últimos años, se ha incrementado las actividades mineras, por lo que ha despertado resistencia social en las poblaciones involucradas. Esta situación afecta las inversiones y por ende afecta su desarrollo y su conflictividad.

En las zonas de conflicto minero los agitadores sociales, han vendido la falsa idea del control, uso, acceso a los recursos naturales. La actividad extractiva en las zonas en conflicto, especialmente de la minería, a pesar de sus problemas es la más importante de Latinoamérica.

Los especialistas en la minería en el mundo, toman como ejemplo el Perú, cuyos proyectos mineros son los mejores del siglo XXI, por que cuentan con estándares internacionales más altas sobre el impacto ambiental. Empero, los conflictos sociales están poniendo en jaque su crecimiento económico. Este escenario demuestra, falta de conocimiento sobre las normas legales concernientes al derecho ambiental, la mala aplicación de un sistema democrático centralista que no toma en cuenta la intercultural de los pueblos, genera desigualdades, han sido aprovechados por los agitadores sociales en beneficio propio.
Esto debido a la escasa participación de los actores en la toma de grandes decisiones.

Los pobladores, por su desconocimiento de las leyes vigentes, se creen dueño de las tierras, cuando las normas dicen todo lo contrario; la propiedad del subsuelo es un derecho distinto a la propiedad del suelo, tanto en el plano legal, como en la práctica son dos derechos diferentes sobre la misma materia. El propietario de la superficie (el suelo) no es propietario del subsuelo, vale decir; de lo que hay bajo sus pies, que pudiera ser suya, peor no lo es.

El Código Civil, detalla esta división irracional de la propiedad. En su artículo $954^{\circ}$ donde establece que "La propiedad del subsuelo no comprende los recursos naturales, los yacimientos y restos arqueológicos, ni otros bienes regidos por leyes especiales". Esto quiere decir que eres propietario de todo lo que hay en el suelo, pero no de lo que hay en el subsuelo. Esta disposición legal, es conveniente precisarlo, analizando la Constitución Política del Perú, que aplicando el criterio de propiedad estatal del subsuelo, establece en su artículo $66^{\circ}$ que "Los recursos naturales, renovables y no renovables, son patrimonio de la nación. El Estado es soberano en su aprovechamiento". En consecuencia ambos instrumentos normativos son expresión de la doctrina jurídi- 
ca denominada "Sistema Dominalista del Estado o de Dominio Inminente del Estado", en el cual el Estado afirma, a priori, que es propietario del subsuelo.

En ese sentido, el reconocimiento legal de los derechos y deberes civiles y políticos, el derecho a la libertad de pensamiento, religión y conciencia, libertad de reunión, igual protección ante la ley, libertad de circulación, derecho a la propiedad, de los pueblos, solo es sustantiva y subjetiva, más no objetiva. Allí radica la dificultad por entender los alcances de las normas en materia derechos ambientales. A raíz de la aplicación de las normas vigentes, las Comunidades Campesinas y Comunidades Nativas, no perciben directamente los beneficios por la explotación de los recursos naturales que se puedan encontrar en el subsuelo de sus propiedades. Esta escasa comunicación, es el pretexto perfecto para que las organizaciones no gubernamentales con fines económicos particulares o por intereses de terceros, aprovechan la ignorancia del pueblo para manipular y lucrar.

Empero se puede acotar a ello, la Ley de Regalías Mineras que establece que las empresas mineras deben efectuar pagos por dicho concepto, los cuales son transferidos a los Gobiernos Regionales, Locales y Universidades, entendiendo que de esta manera se compensa a los pueblos que conforman las áreas de influencia de la actividad minera. Sin embargo todos esos recursos, van a parar al bolsillo de los corruptos, hechos que son caldos de cultivo fácil para los agitadores sociales.

Por otro lado, la importancia de los proyectos mineros, es reconocido y ponderado a nivel internacional, por cuanto sus inversiones En los últimos diez años, han aumentaron en $800 \%$ y la cotización de los principales metales se han triplicado (De Echave y Diez, 2013: 7). En la actualidad involucra una inversión de US\$ $58 \mathrm{mil}$ millones

Para el Ministerio de Economía y el Banco Central de Reserva, la economía peruana depende, entre otros factores, de este tipo de proyectos para su desarrollo. De acuerdo con el Instituto Peruano de Economía, la minería representa el $11 \%$ del Producto Bruto Interno.

Según los especialistas los proyectos mineros de Toromocho, involucra una inversión de US\$ 3, 500 millones; Constancia, con US\$ 1, 400 millones; y la ampliación de Cerro Verde por US\$4 600 millones. Siendo una las mineras emblemáticas,

Las Bambas como un megaproyecto que tendría una dimensión similar al proyecto minero Antamina (departamento de Ancash): con una política minera adecuada, se estima que la producción de cobre en el Perú crecería al menos en $25 \%$, las exportaciones mineras en $30 \%$, mientras que el PBI se incrementaría al 1\%. Por tanto, el caso Las Bambas ofrecería la oportunidad de desarrollo para los pueblos y el país, si existiera voluntad de solucionar los conflictos sociales.

Por otro lado, Las Bambas constituye para el Perú un caso emblemático relevante, en términos de políticas públicas mineras, la licitación del complejo minero, organizado por la Agencia de Promoción de la Inversión (ProInversión) corresponde hoy a la política de privatización del gobierno peruano.

El proyecto minero las Bambas se encuentra ubicado en las provincias sur andinas de Cotabambas (distritos de Coyllurqi, Challhuahuacho y Tambobamba) y Grau (distrito de Progreso) y comprende los yacimientos de Chalcobamba, Ferrobamba, Sulfobamba y Charcas, con un área de concesión minera de 35,000 hectáreas. Puesto que tiene 40,5 toneladas de reservas con más de $2 \%$ de cobre y un potencial mayor a 500 millones de toneladas, el proyecto Las Bambas es calificado como proyecto de clase mundial. Al comprometerse a pagar 121 millones de dólares.

En provincias altas (alrededor de 4000 metros sobre el nivel del mar), los habitantes dependen casi exclusivamente de una economía de autosubistencia orientada a la agricultura (tubérculos) 
y la ganadería. Son 494 las comunidades que se encuentran en los distritos directamente afectados por el proyecto Las Bambas, de las cuales seis tienen terrenos en el perímetro de las concesiones mineras (comunidades de Fuerabamba, Huancuire, pamputa, Chicñahui, Pumamarca y Cconccacca). Apurímac está clasificada como una zona de extrema pobreza y cuenta con Indicadores de Desarrollo Humano (IDH) entre los más bajos del país. Cabe resaltar que en estas zonas, todavía se puede observar una cultura andina viva, con prácticas y creencias tradicionales. Las limitaciones de la participación ciudadana en los procesos de decisión son a la vez vectores de conflictos y elementos bloqueadores en la gestión de los conflictos.

Finalmente, respecto al trabajo de campo, se realizaron entrevistas semi-estructuradas dirigidas a informantes. Además de las entrevistas formales y grabadas, se recolectó mucha información en las conversaciones informales, en la participación en los eventos que se organizaron sobre el tema minero y específicamente sobre Las Bambas, y en la participación en asambleas comunales en la zona (en la comunidad de Fuerabamba).

Cabe precisar, algunas dificultades metodológicas y prácticas respecto al trabajo de campo y las transcripciones de las entrevistas. Una de las ventajas fue que el investigador habla quechua de la zona. Esto facilito el grado de libertad y la fluidez en las entrevistas y se pudo profundizar temas claves que aparecieron a lo largo de las entrevistas y que no se habían contemplado en las guías.

Cabe precisar también, que el trabajo de transcripciones resultó grato, los extractos citados en este informe han sido revisados minuciosamente y corresponden exactamente al discurso de los entrevistados. En una primera parte, se presenta el contexto de los conflictos mineros en el Perú y los rasgos similares que aparecen en varios conflictos mineros del mundo. En una segunda parte, se trata de demostrar los intereses socioeconómicos que intervienen en los conflictos mineros, y los diversos factores culturales existentes.
En el análisis de los conflictos mineros más allá de la esfera socioeconómica, vale decir; mediante el prisma cultural, se abre nuevos horizontes para su prevención y manejo, la distinción establecida por varios autores entre resolución y manejo de conflictos. El manejo no sólo resuelve los problemas ad hoc sino busca una solución analítica a las causas profundas de conflictos, en los términos de John Burton (Burton, 1994). Se trata de visibilizar lo invisible, a saber la dimensión intercultural en los conflictos mineros. Los conflictos mineros presentan una problemática intercultural en el sentido que vuelven evidentes las deficiencias de la ciudadanía en el Perú, en particular el acceso a una ciudadanía respetuosa de las identidades culturales de las poblaciones más marginadas. Por lo tanto, el Estado tendría que garantizar el derecho de sus ciudadanos, no sólo con un marco legal estableciendo principios generales, sino también con un marco institucional que favorezca el ejercicio de la ciudadanía.

Mientras existen algunas herramientas de participación ciudadana, como lo prevé la ley para los Estudios de Impactos Ambientales y las audiencias públicas, o en el marco de acuerdos internacionales como el Convenio 169 de la OIT, en la práctica se limitan a una consulta sin que los resultados se integren siempre a la toma de decisión. Existe una discrepancia de interpretaciones sobre la noción de consulta: para las poblaciones locales, se refiere al derecho de participar en las decisiones vinculadas a las actividades extractivas en sus territorios; en cambio, para los gobiernos, la consulta previa generalmente se refiere una noción más restringida de la participación ciudadana, la potestad de decidir quedando al final en la esfera estatal.

El Banco Mundial planteó el principio de consentimiento libre, previo e informado de los pueblos indígenas para prevenir los conflictos generados por las actividades extractivas de recursos naturales. El principio mencionado es entendido como un derecho colectivo fundamental de las poblaciones indígenas. En efecto, promueve la participación plena y efectiva de los pueblos in- 
dígenas en las decisiones que afectan sus territorios. Convertir este principio en un marco legal obligatorio podría constituir un avance significativo para la protección de los derechos de todos los ciudadanos y por lo tanto, para la prevención de conflictos mineros en el Perú. La implementación de esta herramienta que confiere mayor poder de decisión a las poblaciones locales sobre el destino de sus territorios requiere una reorientación de las políticas públicas peruanas y una redefinición del rol y de las funciones del Estado.

\section{CONCLUSIONES}

Primera conclusión: Luego de revisar los datos bibliográficos y los resultados de las encuestas, sobre las condiciones sociales de los pueblos en las zonas de conflicto minero en el Perú, se concluye que existen evidencias de desconocimiento de las normas legales vigentes de parte de las comunidades que conforman las áreas de influencia minera, el Perú a pesar de ser uno de los países más importantes de Latinoamérica en cuanto a la minería se refiere, se encuentra sumido en uno de los conflictos sociales más sensibles, y delicados, al punto de poner en riesgo su crecimiento económico. Las autoridades nacionales y regionales, no han sabido aplicar los mecanismos de diálogo para la aplicación de las normas constitucionales que pudiera servir para el aprovechamiento de los recursos naturales, asumiendo con mayor responsabilidad los derechos de los pueblos.

Segunda conclusión: Del mismo modo, se concluye que la manera en que se abordan los problemas, repercuten en la reacción violenta de los pueblos. La aplicación a medias de las normas del derecho ambiental relacionado a conflictos sociales no encuentra la cohesión social de los pueblos en las zonas de conflicto minero. Hay desconocimiento de las normas legales vigentes sobre el derecho ambiental de los pobladores, tampoco existe voluntad de preservar el sistema democrático de los pueblos. La socialización de la norma constitucional mediante la consulta previa, puede ser positiva, si es que se aplica con coherencia constitucional y legislativa, cumpliendo la fun- ción social del estado sin desmedro a los derechos identidad natural y cultural de los pueblos.

Tercera conclusión: Asimismo se concluye que la improvisación de las autoridades en la aplicación de las normas del derecho ambiental en las zonas de conflicto minero, ha generado las desigualdades sociales de los pueblos involucrados, que han sido utilizadas hábilmente por los agitadores sociales que desestabilizan toda una estructura de convivencia y tolerancia entre autoridades y el pueblo. Se agrega a ello, la poca participación de las comunidades en la toma de decisiones entre las autoridades competentes y la comunidad, y las instituciones profesionales, llámese colegio de ingenieros, colegio de abogados etc... En temas puntuales como el uso del agua, los bosques y los recursos mineros, temas sensibles que tienen que manejarse dentro del derecho constitucional, al amparo del convenio 169 de la OIT, cuidando el uso de los términos y advirtiendo que no se debe entender como el reconocimiento de derechos que corresponden a los Estados, reconocer sus derechos sobre los recursos existentes en sus territorios, lo que incluye consultarles previamente respecto de su utilización.

Cuarta conclusión: Finalmente se concluye que el conocimiento de los pobladores de las zonas en conflicto sobre las normas del derecho ambiental, es negativa. Por desconocimiento de las leyes, creen ser dueños de las tierras, cuando las normas dicen todo lo contrario; la propiedad del subsuelo es un derecho distinto a la propiedad del suelo, tanto en el plano legal, como en la práctica son dos derechos diferentes sobre la misma materia. El Código Civil, detalla esta división irracional de la propiedad. En su artículo $954^{\circ}$ donde establece que "La propiedad del subsuelo no comprende los recursos naturales, los yacimientos y restos arqueológicos, ni otros bienes regidos por leyes especiales". Esto quiere decir que eres propietario de todo lo que hay en el suelo, pero no de lo hay en el subsuelo. Esta disposición legal, es conveniente precisarlo, analizando la Constitución Política del Perú, que aplicando el criterio de propiedad estatal del sub- 
suelo, establece en su artículo $66^{\circ}$ que "Los recursos naturales, renovables y no renovables, son patrimonio de la nación.

\section{REFERENCIA BIBLIOGRÁFICA}

1. Constitución política del Perú: 1920, 1933, 1979 y 1993. Autor: república del Perú. Edición: congreso de la república del Perú - agosto del 2006.

2. Convenio 169 de la organización internacional del trabajo - resolución legislativa $n^{\circ} 26253$. Autor: organización internacional del trabajo edición: organización internacional del trabajo

3. Los derechos de los pueblos indígenas en el marco de la reforma del estado. Autor: comisión de asuntos indígenas (cai) edición: cámara de diputados de México congreso de la de la unión - 2007.

4. Reglamento de consulta y participación para actividades hidrocarburíferas de Bolivia, decreto supremo n 29033 de 16 de febrero de 2007. Autor: república de Bolivia. Edición: república de Bolivia - 2007.

5. Memoria de la comisión de pueblos andinos amazónicos, afroperuanos, ambiente y ecología del congreso de la república. Autor: comisión de pueblos andinos amazónicos, afroperuanos, ambiente y ecología del congreso de la república. Edición: comisión de pueblos andinos amazónicos, afroperuanos, ambiente y ecología del congreso de la república - julio del 2007.

6. Comunidades en aislamiento voluntario - no contactados autor: derecho ambiente y recursos naturales (dar) edición: bellido ediciones E.I.R.L. - julio 2007.

7. Diagnostico situacional del nivel de cumplimiento de los compromisos asumidos por el gobierno del Perú y la empresa privada en el ámbito del proyecto camisea. Autor: derecho ambiente y recursos naturales (dar) edición: bellido ediciones E.I.R.L. - junio 2007.

8. Diagnostico situacional del nivel de cumplimiento de los compromisos asumidos por el gobierno del Perú y la empresa privada en el ámbito del proyecto del rio corrientes. Autor: racimos de ungurahui. Edición: racimos de ungurahui - diciembre 2007.

9. Manual del grupo de trabajo "racimos de ungurahui" sobre pueblos indígenas. Autor: asociación interétnica de desarrollo de la Amazonia peruana. Edición: asociación interétnica de desarrollo de la Amazonia peruana - 1989.

10. Aporte económico y social de la minería en el Perú. Autor: instituto de ingenieros de minas del Perú. Edición: noviembre del 2007.
11. Una agenda agraria para el desarrollo de la sierra peruana autor: coordinadora de organizaciones campesinas e instituciones agrarias del Perú. Edición: febrero - 2006.

12. Caivano, Roque J., y otros, "mecanismos alternativos para la resolución de conflictos, negociación, conciliación y arbitraje", editor e. Moame drago, primera edición, 1998.

\section{Fisher, Roger y Ury, William, edit. Norma, 1992}

14. Fisher, Roger: "más allá de Maquiavelo, herramientas para afrontar conflictos”. edit. gránica, 1996.

15. Fisher, Roger y Sharp Alan, "el liderazgo lateral, cómo dirigir si usted no es el jefe”, edit. Norma, 1999.

16. Girard, Kathryn y Koch, Susan, "resolución de conflictos en las escuelas”. edit. Gránica, 1997.

17. Jandt, Fred e. y Gillette, Paul: "ganar, ganar negociando, cómo convertir el conflicto en acuerdo", edit. Compañía editorial continental s.a., segunda reimpresión, 1987.

18. Ledesma n., Marianella: "el procedimiento conciliatorio, un enfoque teórico - normativo". edit. Gaceta jurídica s.a., primera edición, 2000.

19. Moore, Christopher,: "el proceso de mediación, métodos prácticos para la resolución de conflictos”, edit. Gráfica, 1995.

20. Ormachea Choque, Iván: "retos y posibilidades de la conciliación en el Perú: primer estudio cualitativo. Propuesta de políticas y lineamientos de acción". Editor: consejo de coordinación judicial. Palacio de justicia. 1998.

21. Oficina técnica de proyectos de cooperación internacional del poder judicial, “acceso a la justicia”, 1997. Ponencias, estudios y comentarios presentados durante el foro “acceso a la justicia” realizado en mayo de 1996.

22. Robbins, Stephen P.: "comportamiento organizacional, conceptos, controversias y aplicaciones", edit. prentice hall, sexta edición 1994.

23. Slaikeu, Karl a.: "para que la sangre no llegue al río", edit. gránica, 1996.

24. Reglamento de la Ley $n^{\circ} 26505$, referida a la inversión privada en el desarrollo de actividades económicas en tierras del territorio nacional y de las comunidades campesinas y nativas (decreto supremo $\mathrm{n}^{\circ}$ 011-97-ag).

25. Ley Forestal y de fauna silvestre, ley $n^{\circ} 27308$, reglamento de la ley forestal y de fauna silvestre, decreto supremo no 014-2001-ag. 
26. La Ley orgánica para el aprovechamiento sostenible de los recursos naturales, dada por ley ${ }^{\circ} 26821$.

27. Ley Orgánica de hidrocarburos, ley n 2622 ;

28. Normas Modificatorias, ley n 26734.

29. Ley $n^{\circ}$ 27377, Ley de actualización de hidrocarburos, texto único ordenado-tuo de la ley orgánica de hidrocarburos, aprobado mediante decreto supremo $\mathrm{n}^{\circ}$ 042-2005-em;

30. Reglamento de las actividades de exploración y explotación de hidrocarburos, aprobado por el decreto supremo no 032-2004-em;

31. Reglamento para la protección ambiental en las actividades de hidrocarburos, aprobado por decreto supremo $\mathrm{n}^{\circ}$ 015-2006-em;

32. Reglamento de participación ciudadana para la realización de actividades energéticas dentro de los procedimientos administrativos de evaluación de los estudios ambientales en el sector energía y minas, resolución ministerial 535-2004-mem-dm. 